\title{
Substituição do arco aórtico sem parada circulatória total: técnicas, táticas e resultados
}

\author{
Fernando Antônio Roquette REIS FILHO*, Luiz Cláudio Moreira LIMA*, \\ Ernesto Lentz da SILVEIRA*, Rodrigo de Castro BERNARDES*
}

RBCCV 44205-551

Reis Filho F A R, Lima L C M, Silveira E L, Bernardes R C - Substituição do arco aórtico sem parada circulatória total: técnicas, táticas e resultados. Rev Bras Cir Cardiovasc 2001; 16(3): 226-35.

RESUMO: Introdução: A despeito dos avanços da cirurgia cardiovascular, das técnicas de circulação extracorpórea e dos métodos de proteção cerebral, a mortalidade nas operações de substituição ou reparo do arco aórtico permanece elevada. As alterações decorrentes da hipotermia profunda e as lesões neurológicas ainda são a maior causa de morbi-mortalidade.

Objetivo: Demonstrar um conjunto de técnicas e táticas cirúrgicas que permite realizar a substituição do arco aórtico sem a necessidade de hipotermia e parada circulatória total e apresentar os resultados alcançados em um grupo de 10 pacientes.

Casuística e Métodos: Dez pacientes do sexo masculino, com idade média de 48,7 anos, foram submetidos a substituição do arco aórtico, utilizando-se hipotermia moderada, canulação arterial em artéria subclávia direita e femoral esquerda, perfusão cerebral seletiva pela artéria subclávia e confecção da anastomose dos vasos arco no primeiro tempo.

Resultados: O tempo médio de perfusão cerebral seletiva foi de 14,1 minutos, de isquemia miocárdica 39,6 minutos e de circulação extracorpórea 98,9 minutos. A temperatura esofágica média foi de $26,6^{\circ} \mathrm{C}\left(24^{\circ} \mathrm{C}\right.$ a $30^{\circ} \mathrm{C}$ ). O tempo médio de internação foi de 18,4 dias (8 a 40 ). Não ocorreram óbitos imediatos ou tardios. Dois pacientes apresentaram confusão mental temporária e um apresentou hemiparesia que foi revertida.

Conclusões: A combinação de técnicas e táticas empregadas permitiu uma efetiva correção das lesões em um campo cirúrgico amplo e seco. Ofereceu uma excelente proteção cerebral sem a necessidade de hipotermia profunda o que diminuiu substancialmente as complicações pósoperatórias.

DESCRITORES: Aorta torácica, transplante. Parada cardíaca induzida, métodos. Circulação extracorpórea, métodos. Hipotermia induzida. Procedimento cirúrgicos cardiovasculares, métodos.

Trabalho realizado no Serviço de Cirurgia Cardiovascular do Hospital Madre Teresa. Belo Horizonte, MG, Brasil. Recebido para publicação em março de 2000.

* Do Serviço de Cirurgia Cardiovascular do Hospital Madre Teresa.

Endereço para correspondência: Fernando Antônio Roquette Reis Filho. Rua Juiz Fora, 1268, sl. 703/707. Bairro Santo Agostinho. Belo Horizonte, MG, Brasil. CEP 30180-061. Telefax: (31) 292-0832. e.mail: froquettereis@ zipmail.com.br 
Reis Filho F A R, Lima L C M, Silveira E L, Bernardes RC - Substituição do arco aórtico sem parada circulatória total: técnicas, táticas e resultados. Rev Bras Cir Cardiovasc 2001; 16(3): 226-35.

TABELA 1

\begin{tabular}{cccc}
\hline & & & DADOS PRÉ-OPERATÓRIOS \\
\hline & SEXO & IDADE & \multicolumn{1}{c}{ DOENÇA } \\
\hline 1 & Masc. & 38 & Dissecção aguda de aorta tipo B com extensão retrograda \\
\hline 2 & Masc. & 57 & Dissecção crônica tipo A com aneurisma de aorta ascendente e arco \\
3 & Masc. & 48 & Aneurisma de arco e aorta descendente após correção de dissecção tipo A \\
4 & Masc. & 47 & Dissecção aguda de aorta tipo A com rotura no arco \\
\hline 5 & Masc. & 39 & Dissecção aguda de aorta tipo B com extensão retrógrada \\
6 & Masc. & 43 & Dissecção crônica tipo B com extensão retrógrada \\
\hline 7 & Masc. & 61 & Infecção de prótese em aorta ascendente com aneurisma de arco \\
8 & Masc. & 61 & Dissecção crônica tipo B com extensão retrógrada \\
\hline 9 & Masc. & 27 & Pseudo-aneurisma traumático de arco aórtico \\
10 & Masc. & 66 & Dissecção subaguda de arco aórtico \\
\hline
\end{tabular}

INTRODUÇÃO

As operações sobre o arco aórtico ainda são um grande desafio porque implicam em uma interrupção do fluxo sangüíneo cerebral. Assim, além de ter que realizar de forma efetiva a reconstrução desta porção da aorta, o cirurgião deve se preocupar em proteger o cérebro para preservar sua vitalidade.

Das técnicas utilizadas a mais difundida é a hipotermia profunda (HP) ${ }^{(1,2)}$, pois esta proporciona ao cirurgião 30 a 40 minutos de parada circulatória total, o que normalmente é suficiente para a correção da doença.

A perfusão seletiva contínua das carótidas talvez seja a técnica mais adequada e fisiológica (3). Esta técnica, porém, exige um aparato complicado e a canulação direta das artérias carótidas, o que não é simples ou isento de complicações.

A retroperfusão cerebral (RPC) surgiu como uma alternativa simples e segura (4-6). Esta técnica, porém, não dispensa a HP. Outras técnicas complementam as anteriores, como a hipotermia tópica craniana, o uso de cerebroplegia (7), drogas como corticóides, barbitúricos e xilocaína ${ }^{(8)}$.

O uso da artéria axilar ou subclávia como linha arterial foi recentemente proposta por BARIBEAU et al. (9) e NERI et al. (10). OKITA et al. (11) utilizaram a canulação da artéria subclávia direita, associada à HP, como forma de proteção cerebral.

Mesmo com estes avanços a mortalidade nas operações de correção das doenças do arco aórtico permanece elevada. As lesões neurológicas e as complicações decorrentes da HP são a maior causa de morbi-mortalidade (12,13). Assim, o objetivo que se persegue é oferecer uma efetiva proteção cerebral com menor tempo de circulação extracorpórea (CEC) e o menor grau de hipotermia possível.

Com este intuito associamos algumas técnicas e táticas que nos permitiram realizar as operações em hipotermia moderada e com menor tempo de CEC. Este trabalho tem por objetivo apresentar estas técnicas e táticas e os resultados obtidos em 10 pacientes consecutivos.

\section{CASUÍSTICA E MÉTODOS}

No período de maio de 1999 a janeiro de 2000, 10 pacientes portadores de doenças crônicas ou agudas envolvendo o arco aórtico foram tratados em nosso Serviço. Os pacientes eram todos do sexo masculino, com idades variando de 27 a 66 anos (média de 48,7 anos). Os dados pré-operatórios estão sumarizados na Tabela 1.

O diagnóstico foi realizado por um ou mais dos seguintes métodos: tomografia computadorizada helicoidal com contraste, aortografia com subtração digital ou ecocardiograma transesofágico.

\section{Técnica Cirúrgica}

Todos os pacientes foram monitorados por pressão intra-arterial em artérias radiais bilateralmente, pressão venosa central através da veia jugular interna direita, sonda vesical de demora, termômetros 
esofágico e retal. Realizamos doppler ultra-som de artéria temporal esquerda para avaliar a perfusão contralateral.

A via de acesso foi a esternotomia mediana. $O$ acesso à artéria subclávia direita foi conseguido por incisão sobre o sulco delto peitoral direito, divulsão do músculo peitoral maior e secção transversal do músculo peitoral menor. A drenagem venosa foi realizada por cânula de duplo estágio em átrio direito.

A CEC foi realizada com oxigenador de membrana (Bard Quantum - ICVR Membrane Oxigenator - Bard Inc., 25 Computer Drive, MA, USA) em hemodiluição total e bomba centrífuga (Centrifugal Pump - Lifestream, Haverhill, MA, USA).

Realizamos hipotermia moderada de 24ㅇ C a $30^{\circ} \mathrm{C}$ (temperatura esofágica). Até atingirmos a temperatura desejada, preparávamos a aorta ascendente, todo o arco aórtico e seus ramos e a aorta descendente proximal. Utilizamos como proteção miocárdica, além da hipotermia tópica e sistêmica, cardioplegia cristalóide gelada, infundida na raiz da aorta, ou diretamente nos óstios coronários. Nos pacientes portadores de aneurisma de arco aórtico e nos portadores de dissecções crônicas e estáveis, canulamos ainda a artéria femoral esquerda através de um desvio na linha arterial (Figura 1).
Utilizamos, em todos os casos, corticosteróide (dexametasona $10 \mathrm{mg}$ ), vasodilatador cerebral (Nimodipina) em infusão contínua e agentes antifibrinolíticos (ácido tranexâmico - 2500mg).

Ao se atingir a temperatura desejada e após a infusão de solução cardioplégica, o tronco braquiocefálico era pinçado. O fluxo arterial não era alterado, sendo autocorrigido pela bomba centrífuga. A aorta ascendente acima dos óstios coronários e a aorta descendente logo abaixo da artéria subclávia esquerda eram separados através de uma incisão transversal e o arco aórtico incisado, todo tecido doente excisado, deixando-se apenas sua porção superior em uma peça única contendo os vasos cervicais. Um enxerto de Dacron (Hemashield Gold Knitted Microvel Double Velour Vascular Graft - Boston Scientific Corporation - Meadox Madicls, Inc.; 112 Bauer Drive - Okland, NJ, USA) de diâmetro adequado e cortado em bisel era anastomosado ao coto do arco contendo os vasos cefálicos (Figura 2).

Completada esta anastomose, o tronco braquioocefálico era despinçado, o ar evacuado e pinçado o enxerto. Assim, estava restabelecida a circulação cerebral (Figura 3). Este período foi denominado tempo de perfusão cerebral seletiva.

Realizávamos, então, a anastomose em aorta descendente, deixando, nos casos de dissecções,

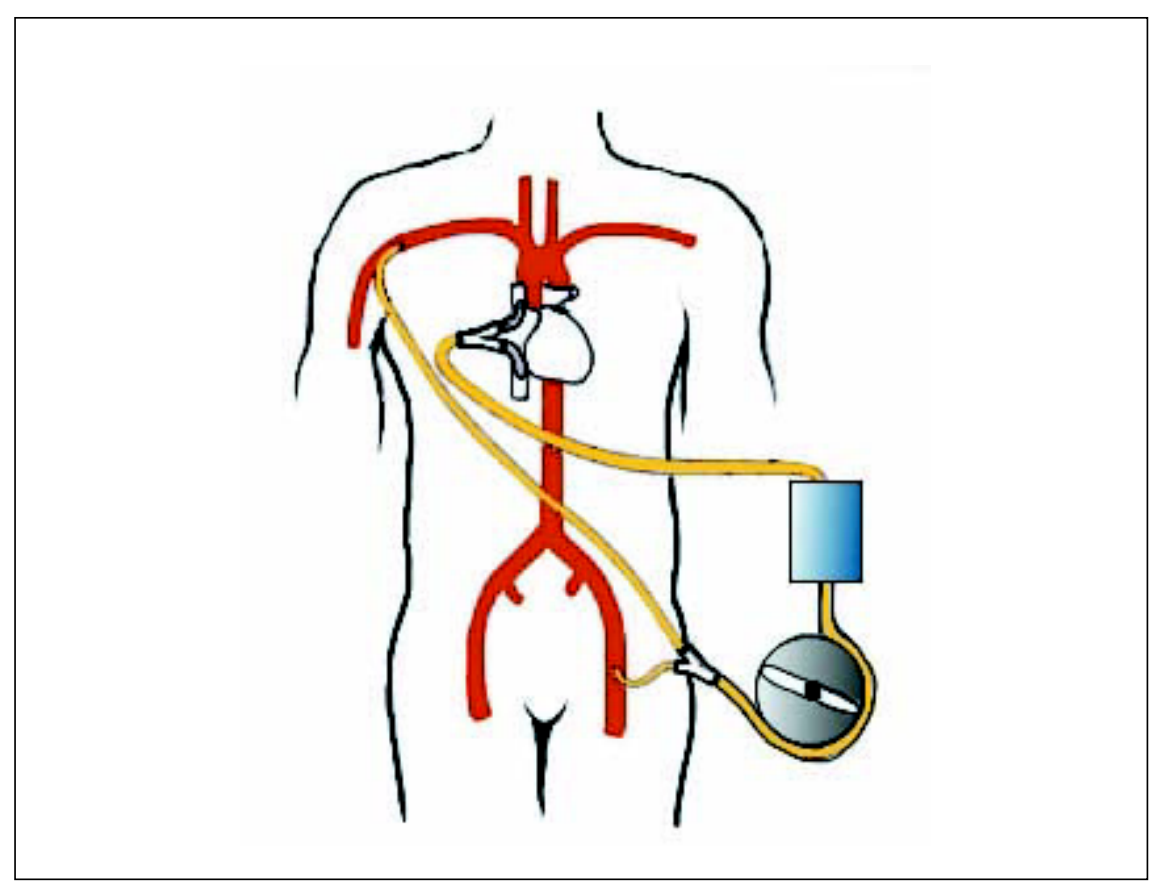

Fig. 1 - Esquema de canulação arterial e venosa. 


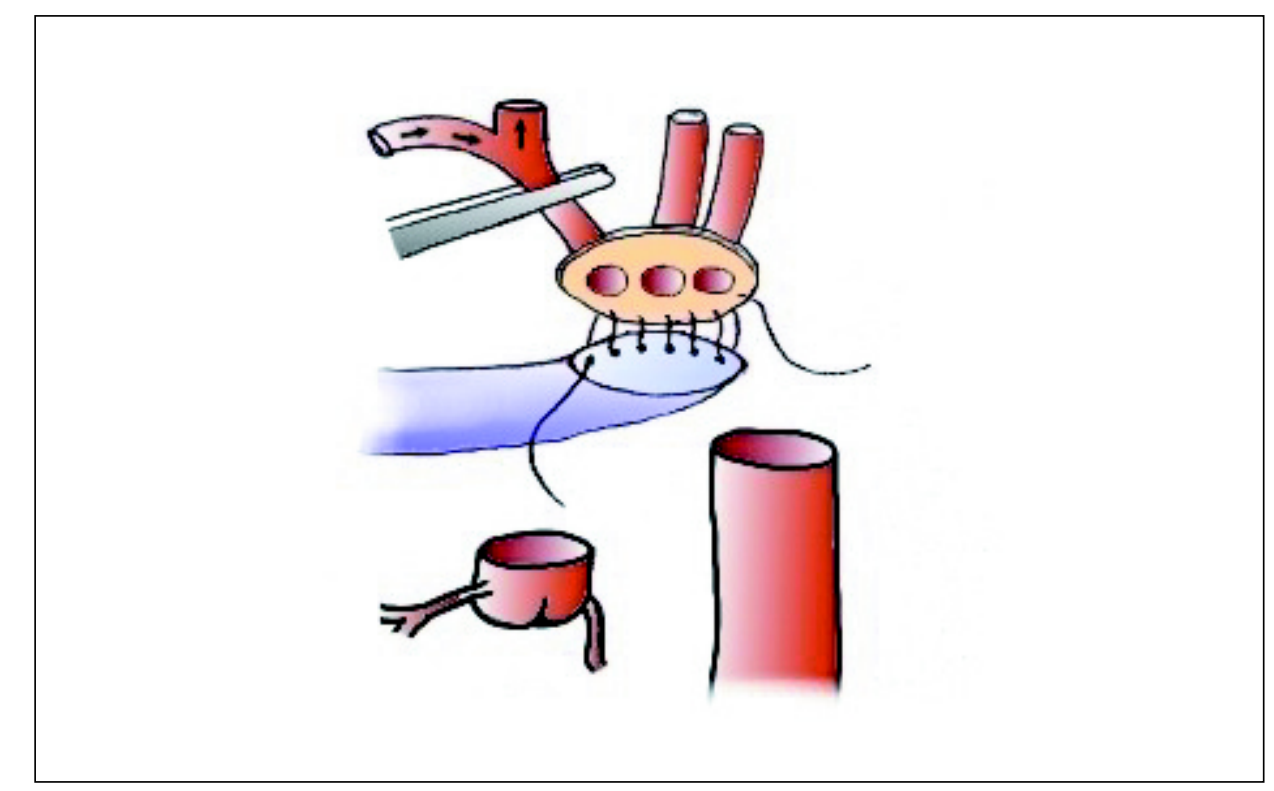

Fig. 2 - Anastomose dos vasos do arco no primeiro tempo com perfusão cerebral via artéria subclávia direita.

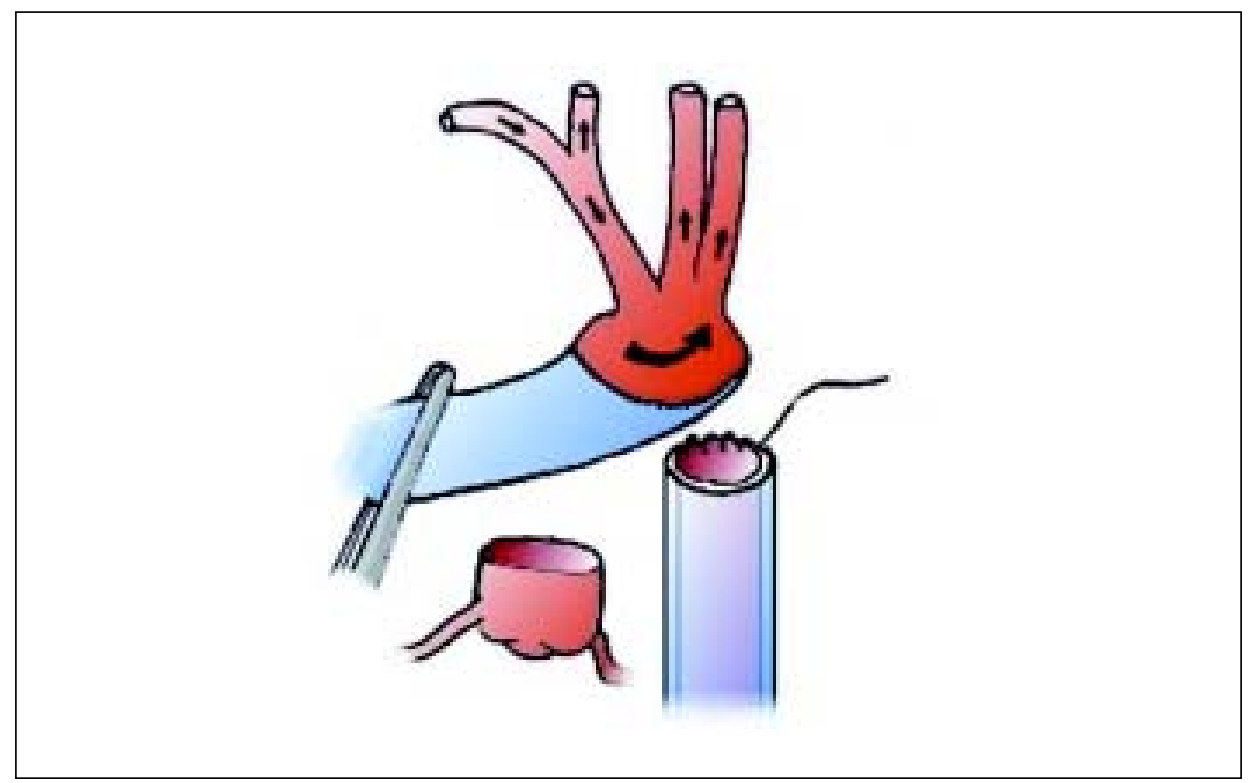

Fig. 3 - Liberação da pinça do tronco braquiocefálico, com normalização do fluxo cerebral e anastomose do enxerto em aorta descendente.

uma pequena "Tromba de Elefante" (14). Após completada esta anastomose, o enxerto era pinçado e a perfusão femoral reiniciada, iniciando também 0 reaquecimento corpóreo de forma lenta (Figura 4). Este período foi denominado tempo de parada circulatória parcial, já que o segmento cefálico já estava sendo perfundido. A outra extremidade do enxerto de aorta descendente era anastomosada término-terminalmente na aorta ascendente, formando um arco.
A seguir, o enxerto dos vasos cervicais era anastomosado término-lateralmente no novo arco, o ar retirado e os pinçamentos liberados. Este período foi denominado tempo de isquemia miocárdica. Completava-se o aquecimento sistêmico até uma temperatura retal de 37ㅇ C (Figura 5). De forma a tornar a operação mais rápida, sempre que possível e necessário, utilizamos o anel intraluminal $(15,16)$ nas anastomoses da aorta ascendente, descendente ou ambas. 


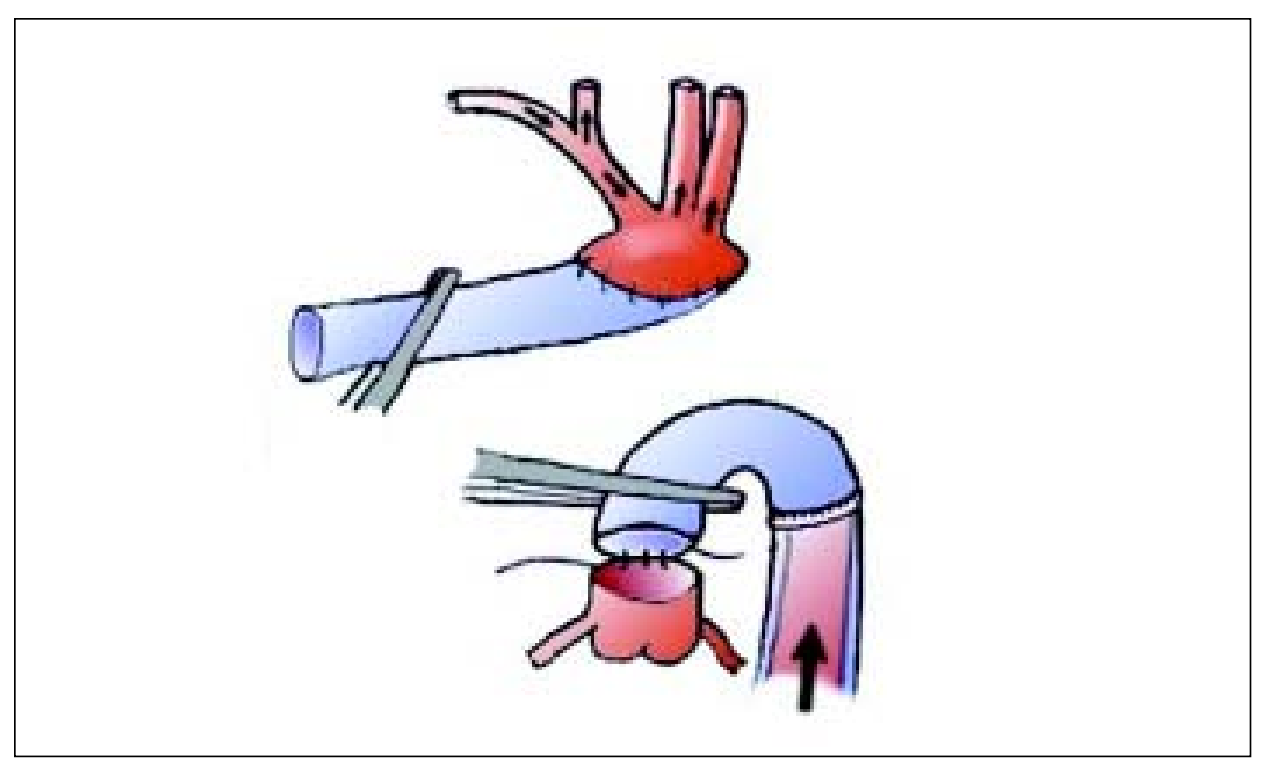

Fig. 4 - Anastomose da outra extremidade do enxerto em aorta ascendente e início da perfusão visceral.

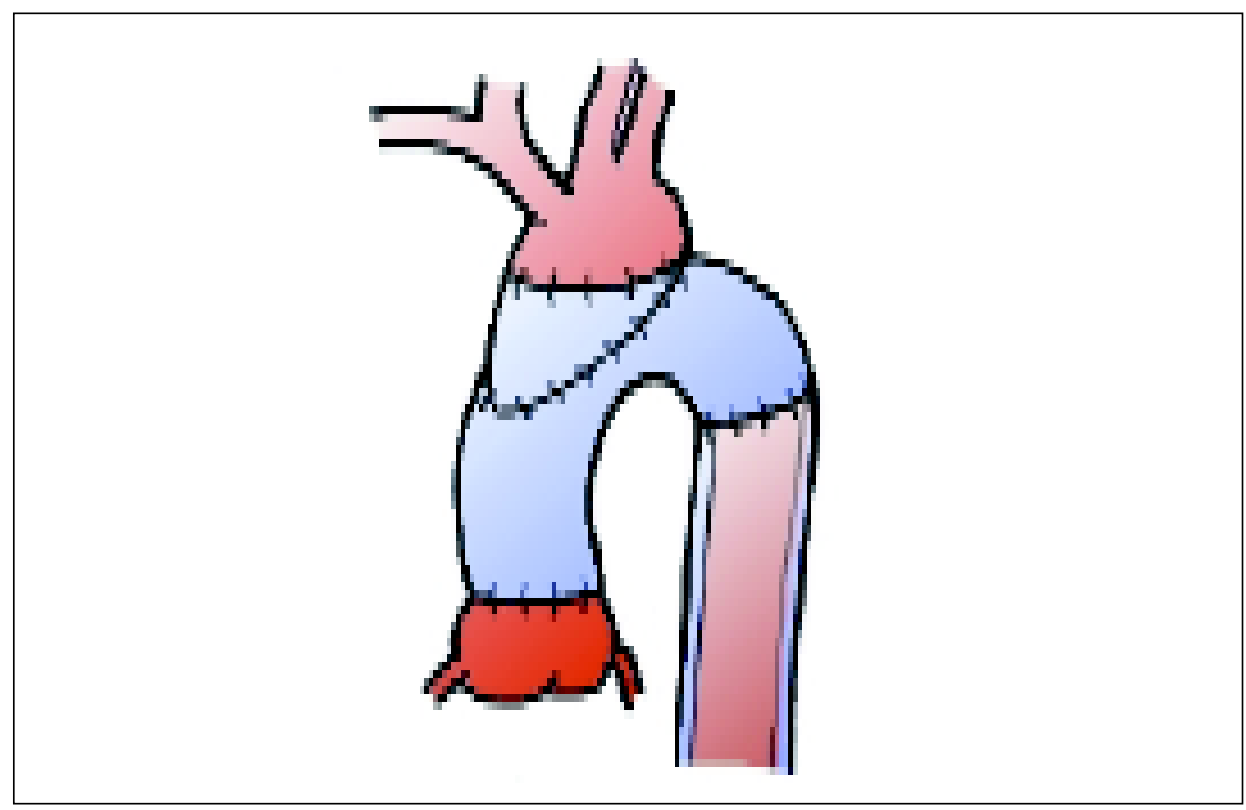

Fig. 5 - Anastomose do enxerto dos vasos cervicais no "novo arco aórtico".

\section{RESULTADOS}

Os dados transoperatórios e pós-operatórios estão discriminados nas Tabelas 2 e 3 . A temperatura demonstrada foi a mínima esofágica alcançada durante o procedimento e o fluxo da bomba centrífuga (fluxo BP) foi a média dos fluxos medidos a cada dois minutos durante a perfusão cerebral seletiva. O uso de hemoderivados está demonstrado por unidades utilizadas.
Dois pacientes apresentaram quadro confusional pós-operatório, sem déficit motor e evoluíram com melhora progressiva apenas com observação. Um paciente (caso 7) evoluiu com hemiparesia esquerda e convulsões focais em perna esquerda, tendo sido induzido coma barbitúrico para controle de convulsões; por isto, este paciente teve tempo de ventilação mecânica elevado (220 horas) e consequentemente pneumonia associada à ventilação mecânica. Este paciente teve alta hospitalar 
Reis Filho F A R, Lima L C M, Silveira E L, Bernardes RC - Substituição do arco aórtico sem parada circulatória total: técnicas, táticas e resultados. Rev Bras Cir Cardiovasc 2001; 16(3): 226-35.

TABELA 2

\begin{tabular}{|c|c|c|c|c|c|c|}
\hline \multirow[b]{2}{*}{ PACIENTE } & \multicolumn{5}{|c|}{ DADOS TRANSOPERATÓRIOS } & \multirow[b]{2}{*}{$\begin{array}{r}\text { T.CEC } \\
\text { (MIN) }\end{array}$} \\
\hline & $\begin{array}{l}\text { TEMPERAT. } \\
\text { ESOFÁGICA }\end{array}$ & $\begin{array}{l}\text { T. PCS } \\
\text { (MIN) }\end{array}$ & $\begin{array}{l}\text { FLUXO BP } \\
\text { (ML/MIN) }\end{array}$ & $\begin{array}{r}\text { T. PCP } \\
\text { (MIN) }\end{array}$ & $\begin{array}{l}\text { T. IM } \\
\text { (MIN) }\end{array}$ & \\
\hline 1 & $25^{\circ} \mathrm{C}$ & 10 & 840 & 20 & 20 & 184 \\
\hline 2 & $24^{\circ} \mathrm{C}$ & 15 & 790 & 32 & 47 & 142 \\
\hline 3 & $24^{\circ} \mathrm{C}$ & 15 & 890 & 40 & 65 & 122 \\
\hline 4 & $24^{\circ} \mathrm{C}$ & 12 & 930 & 32 & 45 & 97 \\
\hline 5 & $26^{\circ} \mathrm{C}$ & 22 & 1610 & 32 & 32 & 65 \\
\hline 6 & $30^{\circ} \mathrm{C}$ & 16 & 1330 & 36 & 57 & 109 \\
\hline 7 & $28^{\circ} \mathrm{C}$ & 14 & 1460 & 17 & 25 & 86 \\
\hline 8 & $28^{\circ} \mathrm{C}$ & 4 & 1560 & 15 & 25 & 65 \\
\hline 9 & $28^{\circ} \mathrm{C}$ & 19 & 1670 & 40 & 51 & 70 \\
\hline 10 & $29 \circ \mathrm{C}$ & 14 & 1520 & 23 & 29 & 49 \\
\hline média & $26,6^{\circ} \mathrm{C}$ & 14,1 & 1260 & 28,7 & 39,6 & 98,9 \\
\hline
\end{tabular}

T. PCS: tempo de perfusão cerebral seletiva; Fluxo BP: fluxo da Biopump durante a perfusão cerebral seletiva; T PCP: tempo de parada circulatória parcial; T IM: tempo de isquemia miocárdica; T CEC: tempo de circulação extracorpórea.

TABELA 3

\begin{tabular}{cccccc}
\hline \multicolumn{7}{c}{ DADOS PÓS-OPERATÓRIOS } \\
\hline PACIENTE & $\begin{array}{c}\text { TEMPO VENTILAÇÃo } \\
\text { MECÂNICA (HORAS) }\end{array}$ & $\begin{array}{c}\text { TEMPO DE } \\
\text { CTI (DIAS) }\end{array}$ & $\begin{array}{c}\text { TEMPO DE } \\
\text { INTERNAÇÕES } \\
\text { DIAS }\end{array}$ & $\begin{array}{c}\text { USO DE } \\
\text { HEMODERIVADOS } \\
\text { (UNIDADES) }\end{array}$ & $\begin{array}{c}\text { COMPLICAÇÕES } \\
\text { NEUROLÓGICAS }\end{array}$ \\
\hline 1 & 36 & 5 & 18 & 15 & -- \\
\hline 2 & 12 & 15 & 23 & 12 & Sim \\
3 & 17 & 2 & 12 & 19 & -- \\
4 & 12 & 9 & 18 & 12 & -- \\
5 & 10 & 24 & 35 & 4 & Sim \\
\hline 6 & 4 & 3 & 10 & 5 & -- \\
7 & 220 & 10 & 40 & 18 & Sim \\
\hline 8 & 6 & 2 & 10 & 4 & -- \\
9 & 4 & 2 & 10 & 2 & - \\
10 & 12 & 2 & 8 & -- & - \\
média & $\mathbf{3 3 , 3}$ & $\mathbf{7 , 4}$ & $\mathbf{1 8 , 4}$ & $\mathbf{9 , 1}$ & - \\
\hline
\end{tabular}

com hemiparesia flácida do membro inferior esquerdo, que evoluiu com recuperação quase total dos movimentos. Não ocorreram outras alterações neurológicas.
Um paciente (caso 3), que foi admitido já com insuficiência renal, apresentou elevação de escórias nitrogenadas, necessitando de diálise peritoneal, mas evoluiu com melhora progressiva. 


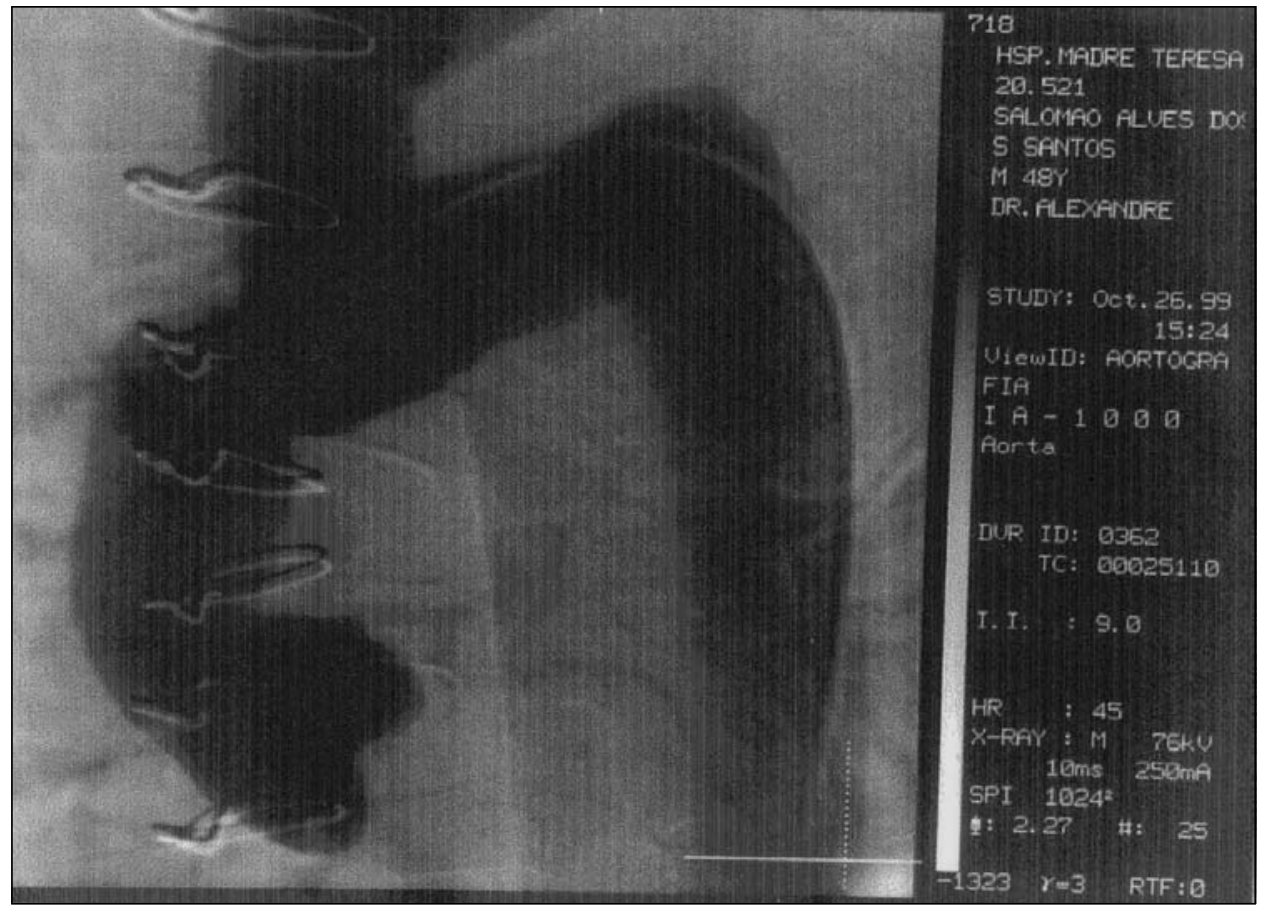

Fig. 6 - Aortografia pós-operatória do mesmo caso.

Este mesmo paciente apresentou importante sangramento no pós-operatório imediato, que foi tratado conservadoramente com infusão de antifibrinolíticos (ácido traneximâmico) e fatores de coagulação (plasma fresco, crioprecipitado e concentrado de plaquetas).

Não ocorreram óbitos na fase hospitalar. Todos os pacientes foram submetidos a reestudo por angiografia, tomografia ou ambos (Figura 6). Em todos os casos consideramos a correção realizada adequada. Um paciente evoluiu com edema do membro superior e outro com alterações de sensibilidade na mão direita. Todos os pacientes encontram-se em controle ambulatorial com boa evolução.

\section{COMENTÁRIOS}

A melhor forma de se proteger um órgão é fornecer sangue oxigenado de forma anterógrada. Inequivocamente, a perfusão seletiva contínua das artérias carótidas é o método mais eficiente de proteção cerebral (17-19). A complexidade do sistema de tubos de CEC, associada à diminuição do campo cirúrgico que a canulação seletiva impõe e ainda o risco de traumatizar o endotélio vascular carotídeo, afastam o cirurgião desta técnica e têm desestimulado sua ampla utilização.
Assim, a parada circulatória total que é possibilitada pela HP proporciona um campo cirúrgico amplo e seco. A HP porém, apesar de relativamente segura, implica em grandes complicações hematológicas e metabólicas decorrentes de uma CEC prolongada, alterações estas eventualmente tão graves que acarretam o óbito do paciente. Além do que após 45 minutos de parada circulatória algum grau de lesão neurológica é quase inevitável $(20,21)$.

A retroperfusão cerebral associada à HP é de fácil realização, não interferindo diretamente no campo cirúrgico. Oferece melhor proteção que a HP isolada $(6,13,22)$, entretanto, parece não fornecer um aporte adequado de oxigênio para a célula nervosa $(18,19,23)$. Sua superioridade (sobre a HP isolada) parece estar associada a sua capacidade de evacuar o sistema arterial de debris e ar e manter a hipotermia (17).

A perfusão seletiva da artéria subclávia, proposta por BARIBEAU et al. (9), NERI et al.(10) e OKITA et al. ${ }^{(11)}$ proporciona um campo cirúrgico amplo, sem sangue e não implica em complexos sistemas de CEC. Previne complicações da canulação femoral como perfusão da falsa luz nas dissecções e embolização retrógrada em pacientes portadores de aterosclerose e aneurismas abdominais. Teoricamente, permite uma adequada perfusão de todo o cérebro através do polígono de Willis e 
Reis Filho F A R, Lima L C M, Silveira E L, Bernardes RC - Substituição do arco aórtico sem parada circulatória total: técnicas, táticas e resultados. Rev Bras Cir Cardiovasc 2001; 16(3): 226-35.

de outros sistemas alternativos colaterais como o vertebral e o carotídeo externo.

Não há trabalho clínico ou experimental comprovando a eficácia da perfusão seletiva da ASD em fornecer adequado fluxo sangüíneo aos dois hemisférios cerebrais. Assim, BARIBEAU et al.(9) e NERI et al. ${ }^{(10)}$ a utilizaram apenas como linha de retorno arterial e OKITA et al. (11) associaram a este método a HP, o que permitiu realizar a correção de forma segura.

Sabemos que o tempo de parada circulatória e o tempo prolongado de CEC são as maiores causas de morbi-mortalidade $(20,21,24)$. Assim, procuramos agrupar técnicas e táticas que permitissem realizar a substituição do arco aórtico com o menor grau de hipotermia e no menor tempo possível.

Para tanto, utilizamos a artéria subclávia direita como linha de retorno arterial, pois assim, pudemos manter uma perfusão cerebral contínua, mesmo que parcial. Realizamos, no primeiro tempo, a anastomose dos vasos cervicais como proposto por LANSMANN \& GRIEPP (25). Na proposição destes autores, logo após esta primeira anastomose, a cânula arterial é realocada para o enxerto de forma a restabelecer o fluxo cerebral. Como a linha arterial já estava instalada na artéria subclávia, só trocamos o pinçamento do tronco braquiocefálico para o enxerto, normalizando, a circulação cerebral e dispensando a canulação da prótese. Assim, economizamos tempo e ganhamos espaço no campo cirúrgico. Em poucos minutos (média de 14,1 minutos), a circulação cerebral estava fisiologicamente restabelecida e o restante da correção podia ser feito com tranqüilidade e segurança. Utilizamos a canulação arterial dupla, nos casos em que isto era permitido, com o intuito de diminuir o tempo de isquemia visceral e permitir um reaquecimento mais precoce.

Utilizamos vasodilatadores cerebrais com o intuito de aumentar o fluxo arterial através dos ramos colaterais e proporcionar melhor perfusão da metade esquerda do cérebro. Sua ação foi comprovada durante a CEC pela observação de alterações no fluxo arterial da bomba centrífuga ocorridas pela modificação de sua taxa de infusão. Os pacientes apresentaram ligeira hipotensão ao início da infusão, que foi facilmente revertida com infusão de volume.

O uso da bomba centrífuga é de extrema importância, pois esta auto-regula seu fluxo de forma inversamente proporcional à resistência que é diretamente influenciada pela hipotermia, assim, não havia necessidade de alterar a velocidade da bomba para atingir um fluxo predeterminado. Alcançamos fluxo de até $1670 \mathrm{ml} / \mathrm{min}$. Com uma média de 750 $\mathrm{ml} / \mathrm{min} / \mathrm{m}^{2} \mathrm{SC}$, bem acima dos $350 \mathrm{ml} / \mathrm{min} / \mathrm{m}^{2} \mathrm{SC}$ normalmente indicados em condições de hipotermia (18). O edema que pode ocorrer quando se trabalha com elevados fluxos em hemodiluição total não foi detectado, pelo menos clinicamente, em nossos pacientes.

Com os primeiros resultados favoráveis, passamos a permitir um menor grau de hipotermia e, nos últimos casos, realizamos hipotermia moderada (28응 $\mathrm{C}$ a $30^{\circ} \mathrm{C}$ esofágica $-30^{\circ} \mathrm{C}$ a $32^{\circ} \mathrm{C}$ retal). Com isto, diminuímos ainda mais o tempo de CEC e as complicações hematológicas e metabólicas, os pacientes acordaram mais rapidamente e, consequentemente, tiveram menor necessidade de hemoderivados, tempo de ventilação mecânica e internação no CTI.

Em nenhum paciente realizamos estudo do polígono de Willis no pré-operatório. Nos primeiros casos realizamos o doppler ultra-som de artéria temporal esquerda durante o período de perfusão cerebral seletiva e detectamos a presença de bom fluxo arterial. Este dado demonstra que é possível proporcionar fluxo arterial contralateral.

Podemos atribuir o baixo índice de complicações neurológicas à faixa etária relativamente baixa dos pacientes, mas também, à existência de outras conexões direita-esquerda cranianas que não o polígono de Willis (sistema vertebral e carotídeo externo).

A morbi-mortalidade em operações de substituição do arco aórtico alcança índices de 0,7\% a 35\% $(4,13)$. O tempo de parada circulatória total é o maior responsável pelas complicações neurológicas ${ }^{(20)}$ e o tempo de CEC que é diretamente relacionado ao grau de hipotermia é isoladamente a maior causa de mortalidade (13). Apesar de termos apresentado uma pequena série, não ocorreram óbitos ou seqüelas neurológicas incapacitantes e o índice de complicações pós-operatórias foi relativamente baixo.

\section{CONCLUSÕES}

O conjunto de técnicas e táticas empregadas possibilitaram uma efetiva proteção cerebral com baixo grau de hipotermia. 0 menor tempo de CEC e o menor grau de hipotermia diminuíram substancialmente as complicações pós-operatórias e, conseqüentemente, a morbimortalidade. Permitiram realizar o procedimento com um campo cirúrgico amplo e seco, sem a necessidade de complexos sistemas de CEC, levando, assim, à completa e efetiva correção das lesões. 
RBCCV 44205-551

Reis Filho F A R, Lima L C M, Silveira E L, Bernardes R C - Substitution of the aortic arch without total circulatory arrest: techniques, tactics and results. Rev Bras Cir Cardiovasc 2001; 16(3): 226-35.

ABSTRACT: Introduction: Despite the development in cardiovascular techniques such as in cardiopulmonary bypass and cerebral protection the mortality in the correlations of the diseases of the aortic arch remains high. Deep hypothermic circulatory arrest and cardiopulmonary bypass times as well as neurological lesions are the major causes of morbi-mortality.

Objective: To show some techniques and tactics that allow the complete substitution of the aortic arch without the need of total circulatory arrest and deep hypothermia, and the results obtained in 10 consecutive patients.

Material and Methods: Ten consecutive patients, all male, with a mean age of 48.7 years, had their aortic arch totally substituted using moderate hypothermia, selective cerebral perfusion via subclavian artery and an arch vessels anastomose performed isolatedly as the first stage.

Results: The mean selective cerebral perfusion time was 14.1 minutes with 39.6 minutes of myocardial ischaemia and 98.9 minutes of cardiopulmonary bypass. The mean esofagic temperature was $26.6^{\circ} \mathrm{C}$ $\left(24^{\circ} \mathrm{C}\right.$ to $30^{\circ} \mathrm{C}$ ). The mean time of hospital stay was 18.4 days ( 8 to 40 ). There were no immediate or late deaths. Two patients had temporary cerebral dysfunction (confusion) and one had hemiparesis, completed recover with time.

Conclusions: The combination of these techniques and tactics allowed an effective correction of the diseases in a wide and dry surgical field. They promoted an excellent cerebral protection without the need of deep hypothermia which decreasing substantially the postoperative complications and mortality.

DESCRIPTORS: Aorta, thoracic, transplantation. Heart arrest, induced, melhods. Extracorporeal circulation, methods. Hypothermia, induced. Cardiovascular surgical procedures, methods.

\section{REFERÊNCIAS BIBLIOGRÁFICAS}

1 Borst H G, Schaudig A, Rudolph W - Arteriovenosous fistula of the aortic arch: repair during deep hypothermia and circulatory arrest. $J$ Thorac Cardiovasc Surg 1964; 48: 443-7.

2 Griepp R B, Stinson E B, Hollingsworth J F, Buehler D - Prosthetic replacement of the aortic arch. $J$ Thorac Cardiovasc Surg 1975; 70: 1051-63.

3 Kazui T, Inoue N, Yamada O, Komatsu S - Selective cerebral perfusion during operation for aneurysms of the aortic arch: a reassessment. Ann Thorac Surg 1992; 53: 109-14

4 Usui A, Abe T, Murase M - Early clinical results of retrograde cerebral perfusion for aortic arch operations in Japan. Ann Thorac Surg 1996; 62: 94-104.

5 Pagano D, Carey J A, Patel R L et al. - Retrograde cerebral perfusion: clinical experience in emergency and elective aortic operations. Ann Thorac Surg 1995; 59: 393-7.

6 Lytle B W, McCarthy P M, Meaney K M, Stewart R W, Cosgrove $3^{\text {rd }} D M$ - Systemic hypothermia and circulatory arrest combined with arterial perfusion of the superior vena cava: effective intraoperative cerebral protection. J Thorac Cardiovasc Surg 1995; 109: 738-43.
7 Robbins R C, Balaban R S, Swain J A - Intermittent hypothermic asanguineous cerebral perfusion (cerebroplegia) protects the brain during prolonged circulatory arrest: a phosphorus 31 nuclear magnetic resonance study. J Thorac Cardiovasc Surg 1990; 99: 878-84.

8 Mitchell S J, Pellet O, Gorman D F - Cerebral protection by lidocaine during cardiac operations. Ann Thorac Surg 1999; 67: 1117-24.

9 Baribeau Y R, Westbrook B M, Charlesworth D C, Maloney $\mathrm{C} \mathrm{T}-$ Arterial inflow via an axillary artery graft for the severely atheromatous aorta. Ann Thorac Surg 1998; 66: 33-7.

10 Neri E, Massetti M, Capannini G et al. - Axillary artery cannulation in type a aortic dissection operations. $J$ Thorac Cardiovasc Surg 1999; 118: 324-9.

11 Okita $\mathrm{Y}$, Minatoya $\mathrm{K}$, Tagusari $\mathrm{O}$ et al. - Prospective randomized comparative study of brain protection in total aortic arch replacement: deep hypothermic circulatory arrest with retrograde cerebral perfusion or selective antegrade cerebral perfusion. In: Annals of the $79^{\text {th }}$ Annual Meeting of the American Association for Thoracic Surgery, New Orleans, LA, USA, 1999, p.132.

12 Kazui T, Kimura N, Yamada O, Komatsu S - Surgical outcome of aortic arch aneurysms using selective cerebral perfusion. Ann Thorac Surg 1994; 57: 904-11. 
Ehrlich M P, Fang W C, Grabenwoger M et al. - Impact of retrograde cerebral perfusion on aortic arch aneurysm repair. J Thorac Cardiovasc Surg 1999; 118: $1026-32$.

14 Borst H G, Walterbusch G, Schaps D - Extensive aortic replacement using "elephant trunk" prosthesis. Thorac Cardiovasc Surg 1983; 31: 37-40.

15 Bernardes R C, Reis Filho F A R, Lima L C M et al. Onze anos de experiência com emprego do anel intraluminal para tratamento das doenças da aorta. Rev Bras Cir Cardiovasc 1999; 14: 200-6.

16 Bernardes R D, Reis Filho F A R, Marino R L et al. Surgical correction of aortic disease using intraluminal, crimped bovine pericardial graft. Ann Thorac Surg 1995; 60 (2 Suppl): S316-21.

17 Katz M G, Khazin V, Steinmetz A et al. - Distribution of cerebral flow using retrograde versus antegrade cerebral perfusion. Ann Thorac Surg 1999; 67: 1065-9.

18 Higami T, Kozawa S, Asada T et al. - Retrograde cerebral perfusion versus selective cerebral perfusion as evaluated by cerebral oxygen saturation during aortic arch reconstruction. Ann Thorac Surg 1999; 67: 1091-6.

19 Filgueiras C L, Winsborrow B, Ye J et al. - A 31 pmagnetic resonance study of antegrade and retrograde cerebral perfusion during aortic arch surgery in pigs. J Thorac Cardiovasc Surg 1995; 110: 55-62.
20 Svensson L G, Crawford E S, Hess H R et al. - Deep hypothermia with circulatory arrest: determinants of stroke and early mortality in 656 patients. $J$ Thorac Cardiovasc Surg 1993; 106: 19-31.

21 Ergin M A, Galla J D, Lansman S L, Quintana C, Bodian C, Griepp R B - Hypothermic circulatory arrest in operations on the thoracic aorta: determinants of operative mortality and neurologic outcome. J Thorac Cardiovasc Surg 1994; 107: 788-99.

22 Safi H J, lliopoulos D C, Gopinath S P et al. - Retrograde cerebral perfusion during profound hypothermia and circulatory arrest in pigs. Ann Thorac Surg 1995; 59: 1107-12.

23 Ye J, Yang L, Del Bigio M R et al. - Neuronal damage after hypothermic circulatory arrest and retrograde cerebral perfusion in the pig. Ann Thorac Surg 1996; 61: 1316-22.

24 Grabenwoger M, Ehrlich M, Cartes-Zumelzu F et al. Surgical treatment of aortic arch aneurysms in profound hypothermia and circulatory arrest. Ann Thorac Surg 1997; 64: 1067-71.

25 Lansman S L \& Griepp R B - Resection of aortic arch aneurysms using hypothermic circulatory arrest. In: Kaiser L R, Kron I L, Spray T L, eds. Mastery of Cardiothoracic Surgery. Philadelphia: LippincottRaven, 1998. p. 472-8. 